\title{
The second conversation: improving end-of-life care conversations
}

\author{
Author: Kimberley St John
}

\section{Aims}

Doctors often find it difficult to engage in conversations about end-of-life care. When these conversations prove their most challenging, they can have a profound and lasting effect on the patient and clinician involved. This can create a culture of fear about initiating future conversations. Isolated education programmes can be effective, but often lack sustainability and reach. Initial conversations broaching the subject of end-oflife care tend to be led by the medical consultant or specialist registrar. It is best practice to offer patients and their advocates a second conversation after imparting significant news. The second conversation suggests this should be led by a junior doctor (foundation or core trainee), affording them the opportunity to build their skills and confidence in communicating end-of-life care issues. Using quality improvement methodology, this project explores what conditions are necessary to embed 'the second conversation' in clinical practice. The learning from this study informs a larger research study led by King's College London.

\section{Methods}

'The second conversation' training tool (Fig 1) was introduced to four clinical teams across three hospital trusts in London. Frequency data capturing successful and missed opportunities are analysed together with confidence questionnaires for trainees and consultant surveys.

\section{Results}

Trainees and consultants found 'the second conversation' useful as an educational tool. Key challenges included clear identification of local opportunities for a 'second conversation', and coordination of staff to allow presence at first conversation and debrief following a 'second conversation'.

\section{Conclusion}

This study has provided understanding of the conditions necessary to successfully embed 'the second conversation' in practice. This study builds on the earlier research findings and will contribute to

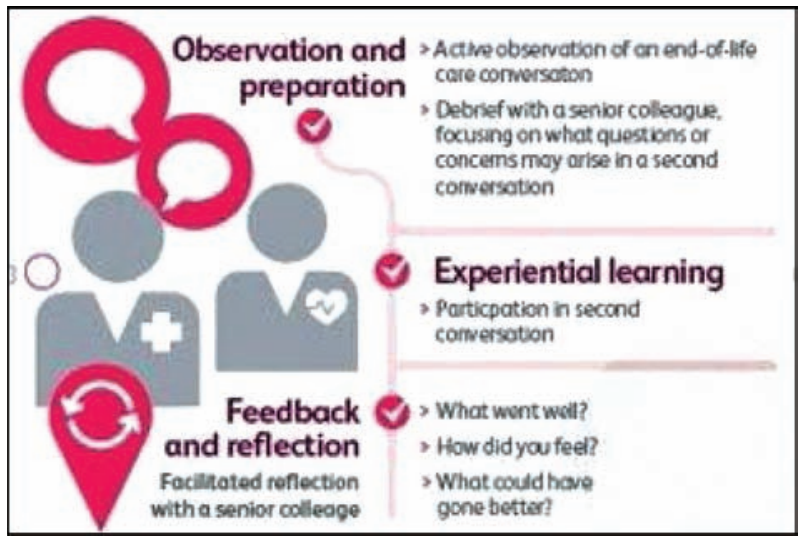

Fig 1. The second conversation training tool.

practical guidance for other teams hoping to embed this innovative training approach.

\section{Conflict of interest statement}

This project is a collaboration between Guy's and St Thomas' NHS Foundation Trust and the Royal College of Physicians. The study lead is currently undertaking a Darzi fellowship which is funded jointly by Health Education England and Guy's and St Thomas' NHS Foundation Trust. No other funding was received to find this study. 